Enhancement of current collection in epitaxial lift-off InAs/GaAs quantum dot thin film solar cell and concentrated photovoltaic study

Tomah Sogabe', Yasushi Shoji, Peter Mulder, John Schermer, Efrain Tamayo, and Yoshitaka Okada

Citation: Appl. Phys. Lett. 105, 113904 (2014); doi: 10.1063/1.4896114

View online: http://dx.doi.org/10.1063/1.4896114

View Table of Contents: http://aip.scitation.org/toc/apl/105/11

Published by the American Institute of Physics






\title{
Enhancement of current collection in epitaxial lift-off InAs/GaAs quantum dot thin film solar cell and concentrated photovoltaic study
}

\author{
Tomah Sogabe, ${ }^{1, a)}$ Yasushi Shoji, ${ }^{1}$ Peter Mulder, ${ }^{2}$ John Schermer, ${ }^{2}$ Efrain Tamayo, ${ }^{1}$ \\ and Yoshitaka Okada ${ }^{1}$ \\ ${ }^{1}$ Research Center for Advanced Science and Technology (RCAST), The University of Tokyo, 4-6-1 Komaba, \\ Meguro-ku, Tokyo 153-8504, Japan \\ ${ }^{2}$ Institute for Molecules and Materials, Radboud University Nijmegen, Heyendaalseweg 135, \\ 6525 AJ Nijmegen, The Netherlands
}

(Received 18 June 2014; accepted 7 September 2014; published online 18 September 2014)

\begin{abstract}
We report the fabrication of a thin film InAs/GaAs quantum dot solar cell (QD cell) by applying epitaxial lift-off (ELO) approach to the GaAs substrate. We confirmed significant current collection enhancement $\left(\sim 0.91 \mathrm{~mA} / \mathrm{cm}^{2}\right)$ in the ELO-InAs QD cell within the wavelength range of $700 \mathrm{~nm}-900 \mathrm{~nm}$ when compared to the ELO-GaAs control cell. This is almost six times of the subGaAs bandgap current collection $\left(\sim 0.16 \mathrm{~mA} / \mathrm{cm}^{2}\right)$ from the wavelength range of $900 \mathrm{~nm}$ and beyond, we also confirmed the ELO induced resonance cavity effect was able to increase the solar cell efficiency by increasing both the short circuit current and open voltage. The electric field intensity of the resonance cavity formed in the ELO film between the Au back reflector and the GaAs front contact layer was analyzed in detail by finite-differential time-domain (FDTD) simulation. We found that the calculated current collection enhancement within the wavelength range of $700 \mathrm{~nm}-900 \mathrm{~nm}$ was strongly influenced by the size and shape of InAs QD. In addition, we performed concentrated light photovoltaic study and analyzed the effect of intermediate states on the open voltage under varied concentrated light intensity for the ELO-InAs QD cell. (C) 2014 AIP Publishing LLC. [http://dx.doi.org/10.1063/1.4896114]
\end{abstract}

The intermediate band solar cell (IBSC) has recently drawn a lot of attention due to its reported theoretical efficiency limit of $63.2 \%$ under radiative recombination and maximum light concentration. ${ }^{1}$ A major feature of the IBSC is that it ensures the solar cell to harvest more sunlight in a wider wavelength range of the solar spectrum through two step photon absorption via the intermediate band (IB) without deteriorating the output voltage if the IB is made electrically isolated from the valence band (VB) and conduction band (CB) of the host material. ${ }^{2}$ Great efforts have been devoted to fabricate IBSC with the IB formed from a quantum dot (QD) superlattice. ${ }^{1}$ Self-assembled InAs QD arrays fabricated by molecular beam epitaxy (MBE) have been widely studied as a building block to create the IB in the GaAs host material. ${ }^{3}$ For an InAs/GaAs QD cell, the substrate (here, GaAs) is one of the most significant cost contributors to the overall system. The cost of these cells could potentially be reduced through the realization of an ultra-thin film solar cell by removal and re-use of the expensive substrate using the epitaxial lift-off (ELO) method. ${ }^{4}$ An additional advantage of an ELO thin film solar cell is that it can effectively increase the path of photons inside the thin film and an optical resonance cavity is expected to be formed to enhance the light absorption. Recently, Bennett et al. ${ }^{5}$ reported successful fabrication of ELO based InAs/GaAs strain-balanced QD solar cells, which exhibited an enhanced sub-GaAs bandgap current collection extending into the near infrared wavelength beyond $900 \mathrm{~nm}$.


sogabe@mbe.rcast.u-tokyo.ac.jp
}

In this paper, we report the fabrication of a thin film InAs/GaAs QD cell by applying the ELO approach. Except the enhanced sub-GaAs bandgap current collection from $900 \mathrm{~nm}$ and beyond due to the light absorption from the InAs QD, we observed a significant current collection enhancement in the ELO-InAs QD cell within the wavelength range of $700 \mathrm{~nm}-900 \mathrm{~nm}$ when compared to the ELO-GaAs control cell. We also confirmed that the resonance cavity effect is able to increase both the short circuit current and open circuit voltage. The electrical field intensity and the absorption behavior inside the ELO induced resonance cavity was analyzed in detail by finite-differential time-domain (FDTD) simulations. Furthermore, we conducted a photovoltaic study under concentrated light illumination and analyzed the effect of intermediates states on the performance of the ELO-InAs QD cell.

The ELO approach used here is similar to our previous reports ${ }^{4}$ and the detailed mechanism of the etching process during ELO can be found in literature. ${ }^{6}$ Epilayers for both GaAs control cells and InAs/GaAs QD cells were fabricated by adopting a $p$ - $i$ - $n$ cell structure on a n-type $\operatorname{GaAs}(100) \pm$ $0.1^{\circ}$ substrate using MBE. We have prepared four samples: a GaAs control cell and an InAs/GaAs QD cell both fabricated on their native GaAs substrate; an ELO GaAs control cell and an ELO InAs/GaAs QD cell. The sketch of the InAs QD cell prepared on its GaAs substrate and the thin film ELOInAs QD cell on a supporting foreign carrier are shown in Fig. 1. The device processing procedures were similar to those reported before. ${ }^{6}$ The current-voltage (I-V) and external quantum efficiency (EQE) of the samples were measured at room temperature. All characterizations were performed for the samples without anti-reflection coating. 

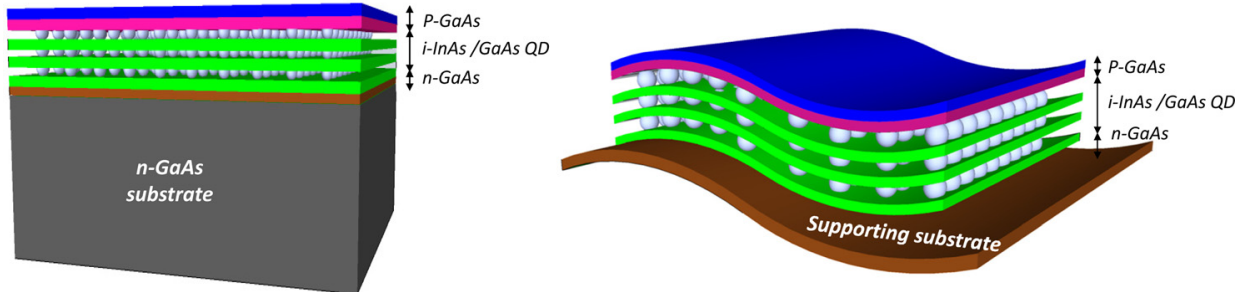

FIG. 1. Illustration of the InAs QD cell on its native GaAs substrate (left) and the thin film ELO-InAs QD cell structure on a flexible foreign carrier (right).
Fig. 2(a) shows an atomic force microscopy (AFM) image of a densely packed InAs QD array with uniform size. The areal density of the InAs QD was estimated to be $\sim 1 \times 10^{10} / \mathrm{cm}^{2}$ with the base length and height of the dots being approximately are $25 \mathrm{~nm}$ and $4 \mathrm{~nm}$, respectively. Fig. 2(b) shows photos of completed ELO-InAs QD cells with different sample area: $1 \mathrm{~cm}^{2}(6 \mathrm{pcs})$ and $0.169 \mathrm{~cm}^{2}(6 \mathrm{pcs})$. The EQE measurement results are presented in Fig. 2(c) for the ELO-GaAs control cell and the ELO-InAs QD cell. It clearly shows here that the QD cell exhibits extended spectral response in the wavelength range beyond $900 \mathrm{~nm}$, which is the absorption edge for the GaAs control cell. The enhanced absorption corresponds to a current collection density of $\sim 0.16 \mathrm{~mA} / \mathrm{cm}^{2}$ converted under AM1.5 and 1 sun illumination conditions. The extended spectral response is due to the light absorption via the InAs QD states, which is consistent with the results reported before. ${ }^{5}$ In addition, it is noticed from Fig. 2(c) that the spectral responses of the thin film and substrate cells have different signatures in the wavelength range: $400 \mathrm{~nm}-900 \mathrm{~nm}$. In the short wavelength range up to $700 \mathrm{~nm}$, which is mainly corresponding to the emitter absorption, we found that the spectral response is lower in the ELO-InAs QD cell when compared to the ELO-GaAs control cell. We have attributed the reduced spectral response to absorption loss caused by incomplete etching of the GaAs contact layers during device processing of the ELO-InAs QD cell. However, contrary to the lower spectral response between $400 \mathrm{~nm}$ and $700 \mathrm{~nm}$, the ELO-InAs QD cell showed much higher intensities of spectral response in the wavelength range from $700 \mathrm{~nm}$ to $900 \mathrm{~nm}$. This increase corresponds to a current collection density of $\sim 0.91 \mathrm{~mA} / \mathrm{cm}^{2}$ (AM1.5 and 1 sun). The multiple resonance peaks in the EQE spectra are due to Fabry-Perot interference and will be analyzed in detail later in the FDTD simulation.

We also performed I-V measurements on the four cells of which the results are shown in Fig. 2(d). We found that the ELO-GaAs control cell presented the best performance among the four samples. Compared to the GaAs control cell on the substrate, the short circuit current density $\left(\mathrm{J}_{\mathrm{sc}}\right)$ of ELO-GaAs control cell has increased more than $1.5 \mathrm{~mA} / \mathrm{cm}^{2}$ and the open circuit voltage $\left(\mathrm{V}_{\mathrm{oc}}\right)$ has increased about $0.02 \mathrm{~V}$. The increase of $\mathrm{J}_{\mathrm{sc}}$ for ELO solar cell device is an expected result because of the light trapping effect which significantly increases the light absorption. The observed $\mathrm{J}_{\mathrm{sc}}$ increase is also consistent with our previous works. ${ }^{7}$ The current and voltage gains observed for the ELO-GaAs cell here demonstrates the effectiveness of applying the ELO technique to not only reduce the device cost but also to further boost the cell efficiency. We must mention here that the fact that the ELO-InAs QD cell showed quite poor $\mathrm{J}_{\mathrm{sc}}$ is mainly due to imperfect etching of the GaAs contact layer during device processing, as was also revealed in the EQE measurements shown in Fig. 2(c). Meanwhile, the $\mathrm{V}_{\text {oc }}$ of ELO-InAs QD cell was also found lower than that of GaAs control cell, (a)

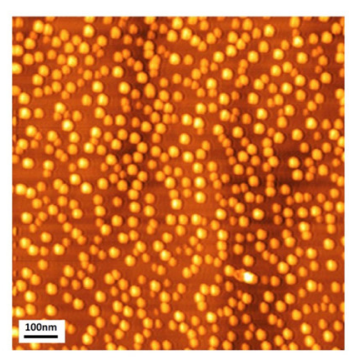

(b)

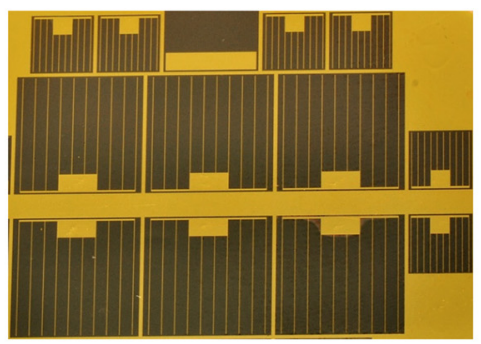

(c)
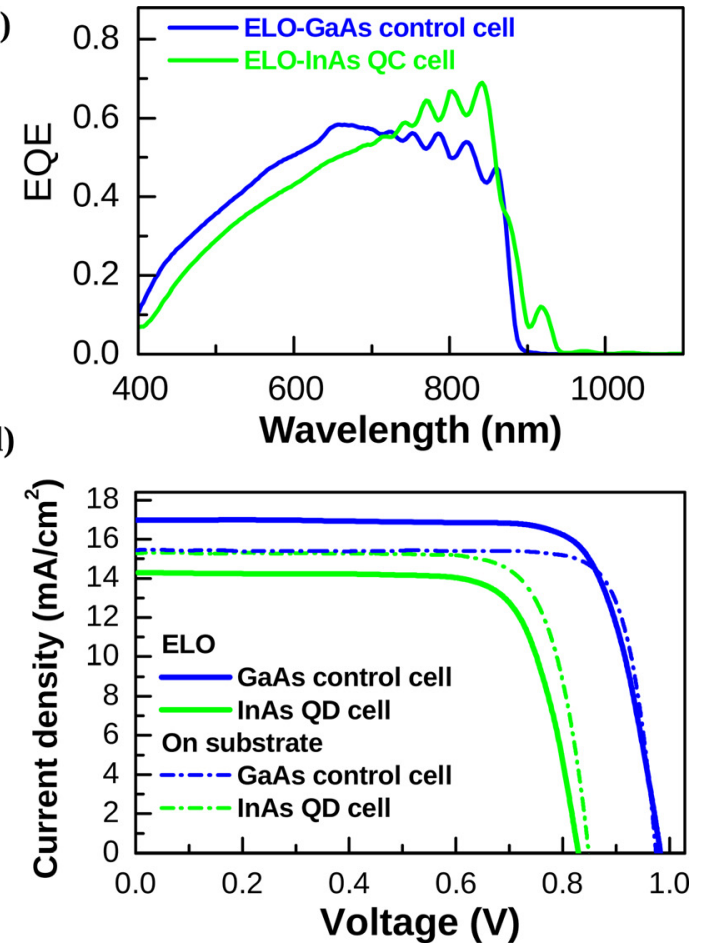

FIG. 2. (a) AFM image of the InAs QD layer. (b) Photo of a set of completed ELO solar cells on a thin film of about $4 \times 3 \mathrm{~cm}^{2}$. (c) EQE of the GaAs control cell on its GaAs substrate and the ELO-InAs QD cell. (d) I-V curves for all investigated cell structures. 




(b)

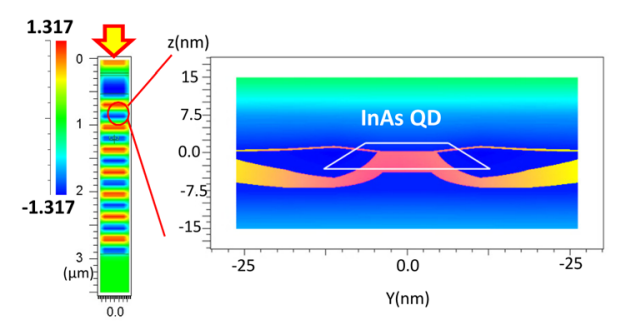

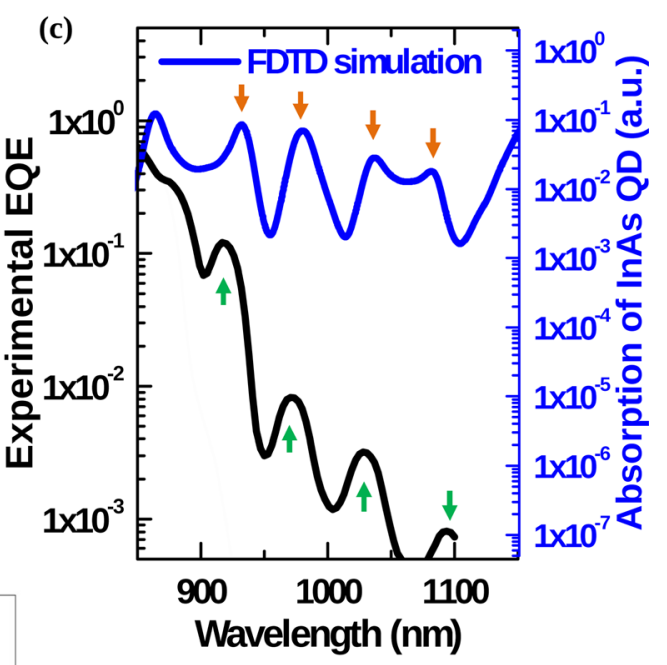

FIG. 3. (a) Schematic representation of the cell structure for the FDTD simulations. (b) Simulated electric field distribution along the $\mathrm{z}$-axis and a close-up of the light absorption around an InAs QD region. (c) Comparison between the experimental EQE analysis (green and blue lines) and FDTD simulations (orange line). which is most likely related to the inherent radiative recombination issue due to the presence of InAs QD. We have found from our previous dark I-V experimental data on InAs QDSC on GaAs substrate that the dark radiative saturation current: $\mathrm{J}_{0, \mathrm{r}}$ was several orders of magnitude larger than the dark non-radiative saturation current: $\mathrm{J}_{0, \mathrm{nr}} \cdot{ }^{8}$ More detailed discussion will be presented in a forthcoming publication on the dark I-V analysis of ELO-InAs QDSC.

In order to get deeper understanding of the optical interference inside the resonant optical cavity and to reproduce the multiple resonant peaks observed in the EQE graphs of the ELO thin film cells, FullWAVE ${ }^{\mathrm{TM}}$ (RSoft Design Group, Inc.) was used to calculate the electromagnetic field as a function of time and space in response to a given excitation. FullWAVE is based on the FDTD method and on the Yee's mesh. Fig. 3(a) shows the FDTD simulation model including the device structure of the GaAs solar cell with embedded InAs QDs and an Au back mirror. The thickness of the GaAs slab is $2400 \mathrm{~nm}$. The bottom of the first InAs QD layer is placed at $650 \mathrm{~nm}$ from the top surface of the Air/GaAs interface (see Fig. 3(a)). The shape of the quantum dots was simulated with a $25 \mathrm{~nm} \times 25 \mathrm{~nm}$ based pyramid structure, truncated at the half height of its $10 \mathrm{~nm}$ top (see Fig. 3(b)). Periodic boundary conditions were used along the X- and Yaxis and perfectly matched layer (PML) boundaries along the Z-axis. In addition, the reflectance, which evidences the back mirror and interferences effects, was also monitored. Light absorption in the GaAs and InAs QD regions were calculated. The optical absorption coefficient of the InAs QDs was calculated using the Fourier transformed 8 -band $k \cdot p$ method. ${ }^{9}$

Fig. 3(b) shows the electric field distribution inside the GaAs slab and around the InAs QD region. The light is trapped due to the low absorption in GaAs for wavelengths above $900 \mathrm{~nm}$. The light trapping creates interferences that enhance the electric field. For this reason, the maximum in the amplitude bar exceeds 1.0. The right hand side of Fig. 3(b) shows that the field is modified around the InAs QD indicating that the enhanced electric field has induced enhanced absorption in the QD layers. Fig. 3(c) shows the comparison between the multiple resonance peaks in the simulated absorption spectrum and the experimental EQE results. The positions of peak maxima (labeled by arrows) show good agreement between the simulation and the experimental results for the ELO InAs QD cell in the absorption region between $900 \mathrm{~nm}$ and $1150 \mathrm{~nm}$.

The absorption between $700 \mathrm{~nm}$ and $900 \mathrm{~nm}$ for both the ELO GaAs control cell and the ELO InAs QD cell were simulated in more detail. Fig. 4(a) shows the experimental spectral response results for the two ELO cells. It shows that the ELO-InAs QD cell exhibits a much larger spectral response, corresponding to a current density increase of $\sim 0.91 \mathrm{~mA} / \mathrm{cm}^{2}$ compared to the ELO-GaAs control cell. Fig. 4(b) shows the simulated results of the absorption inside the GaAs slab and the InAs QD regions. It indicates that the FDTD results based on the ELO-InAs QD model has a much stronger absorption than those of the ELO-GaAs control cell model. We also found the anti-phase of the multiple interference peaks between the InAs QD cell and the GaAs control (a)

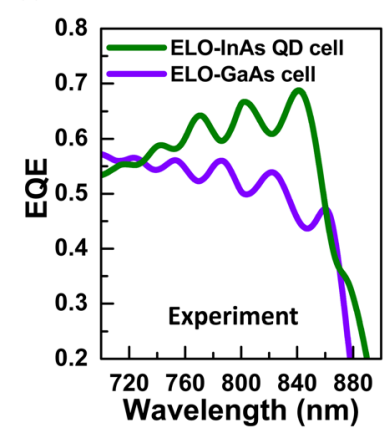

(b)

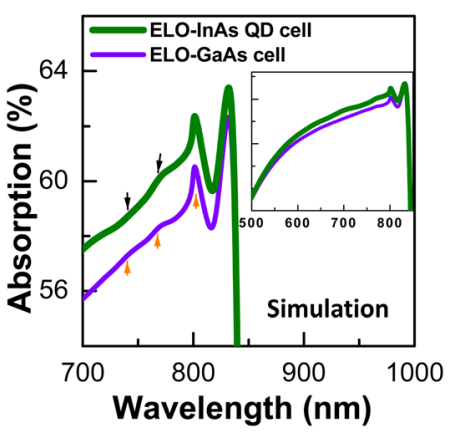

FIG. 4. (a) Experimental analysis of the EQE within the wavelength range of $700 \mathrm{~nm}-900 \mathrm{~nm}$. (b) FDTD simulations of the absorption in the cell structures. Inset shows the absorption in the wavelength range between $500 \mathrm{~nm}$ and $850 \mathrm{~nm}$. 
cell observed in the experiment were reproduced in the simulations (see the position of the arrows). It is interesting to notice here that the enhanced absorption in the FDTD simulation for the ELO-InAs QD cell actually started from $500 \mathrm{~nm}$ as shown in the inset of Fig. 4(b). This indicates that current collection in the ELO-QD cell can potentially be further improved and the maximum current density enhancement can even be larger than the present value of $\sim 0.91 \mathrm{~mA} / \mathrm{cm}^{2}$.

In order to investigate more details about the effect of the InAs QD on the current collection enhancement between $700 \mathrm{~nm}$ and $900 \mathrm{~nm}$, we performed FDTD by varying the size and the shape of InAs QD (see Fig. 5). It is seen here that the absorption, which is proportional to the $\mathrm{EQE}$, increases with the increase of the QD size from $25 \mathrm{~nm}$ to $100 \mathrm{~nm}$. But the increase saturates if the QD size is further increased beyond $100 \mathrm{~nm}$. We can also see the profile of the absorption changes dramatically in the wavelength range below $820 \mathrm{~nm}$ if the shape of the QD is set to be a regular tetrahedron with the base length of $200 \mathrm{~nm}$. We speculate that the ten layers of InAs QD superlattice have acted as an effective light scattering source at the wavelength region studied. Similar photocurrent enhancement below $900 \mathrm{~nm}$ in an InAs/GaAs QDSC fabricated on a GaAs substrate was reported, ${ }^{10,11}$ where the scattering of the electron and hole wave functions by the potential wells of embedded InAs QDs was attributed to greatly enhance observed photocurrent below $900 \mathrm{~nm}$. In the future, we will conduct more detailed investigation by tracing the light path inside the optical cavity to gain much deeper insight on the interplay among the light scattering of QD and occurrence of the current enhancement below $900 \mathrm{~nm}$ as well as its influence on the light absorption of QDs beyond $900 \mathrm{~nm}$.

At last, we present the I-V measurements results for the ELO InAs QD cell under different concentration ratios X, as shown in Fig. 6(a). For the ELO-InAs QD cell, we have reached the highest efficiency of $10.0 \%$ under 52 suns. The fill factor dropped dramatically after the concentration ratio went up beyond 100 suns (see the orange and blue lines in Fig. 6(a)). We attribute the efficiency reduction at high concentration to the non-optimized configuration of the front contact grid electrode used in this study. Fig. 6(b) shows the dependence of $\mathrm{V}_{\mathrm{oc}}$ on $\mathrm{X}$. As a reference, we also plotted the calculated $\mathrm{V}_{\mathrm{oc}}(\mathrm{X}) \sim \mathrm{X}$ for an ideal single junction solar cell

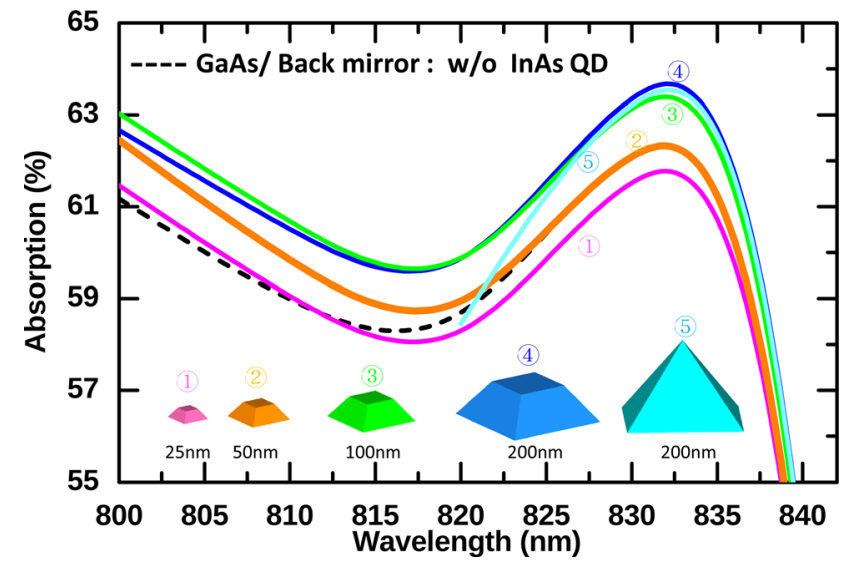

FIG. 5. FDTD simulations of the absorption in the cell structures including the effects from the InAs QD size and shape.
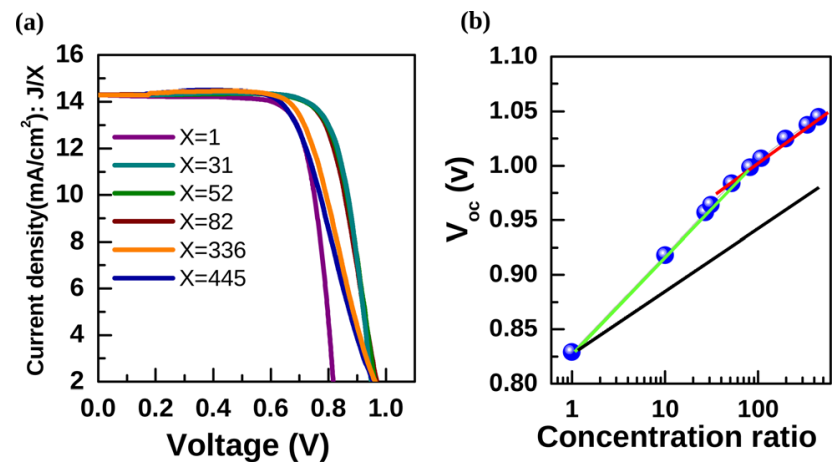

FIG. 6. (a) I-V measurements for the ELO-InAs QD cell under different light concentration ratios. (b) Dependence of $\mathrm{V}_{\mathrm{oc}}$ on concentration ratio $\mathrm{X}$.

(black line): $V_{\mathrm{oc}}(X)=\left[V_{\mathrm{oc}}(1\right.$ sun $\left.)+0.025 \ln (X)\right]$. It is shown here that there exist two different increase rates of $\mathrm{dV}_{\mathrm{oc}}(\mathrm{X}) /$ $\mathrm{dX}$ in the ELO-QD cell: an accelerated increase rate region (green line) for $\mathrm{X}$ below 100 suns and a regular increase rate region (red line) for $\mathrm{X}$ above 100 suns, The regular increase rate resembles the voltage increase rate of the ideal single junction solar cell (black line). The two increase rates obtained here are in good agreement with theoretical calculations involving the typical features of an intermediate band cell, ${ }^{12,13}$ indicating that the intermediate band is functioning as expected in the ELO-InAs QD cell.

In conclusion, we have fabricated an ELO based thin film InAs QD cell. Except for the enhanced sub-GaAs bandgap current density collection $\left(\sim 0.16 \mathrm{~mA} / \mathrm{cm}^{2}\right)$ beyond $900 \mathrm{~nm}$, we confirmed a significant current density collection enhancement $\left(\sim 0.91 \mathrm{~mA} / \mathrm{cm}^{2}\right)$ in the ELO-InAs QD cell within the wavelength range of $700 \mathrm{~nm}-900 \mathrm{~nm}$. The multiple resonance peaks appearing in the EQE results for both the ELO-GaAs control cell and the ELO-InAs QD cell were studied by FDTD simulations. It was shown that the resonance peaks can be well reproduced by calculating the electric field distribution in the Fabry-Perot resonance cavity. From the FDTD simulation results, we claim that the ten layers of InAs QD superlattice are responsible for occurrence of the current enhancement within $700 \mathrm{~nm}-900 \mathrm{~nm}$ wavelength range while acting as an effective light scattering source. Additionally, we performed I-V measurements on the ELO-InAs QD cell under concentrated light and verified the intermediate band features for the ELO-InAs QD cell.

The authors gratefully acknowledge the funding from New Energy and Industrial Technology Development Organization (NEDO), and the Ministry of Economy, Trade and Industry (METI), Japan, and support from the European fund for regional development (EFRO).

\footnotetext{
${ }^{1}$ A. Luque and A. Martí, Phys. Rev. Lett. 78, 5014 (1997).

${ }^{2}$ A. Luque, A. Martí, and C. Stanley, Nat. Photonics 6, 146 (2012).

${ }^{3}$ R. Oshima, A. Takata, and Y. Okada, Appl. Phys. Lett. 93, 083111 (2008).

${ }^{4}$ G. J. Bauhuis, P. Mulder, E. J. Haverkamp, J. J. Schermer, E. Bongers, G. Oomen, W. Kostler, and G. Strobl, Prog. Photovoltaics: Res. Appl. 18, 155 (2010).

${ }^{5}$ M. F. Bennett, Z. S. Bittner, D. V. Forbes, S. R. Tatavarti, S. P. Ahrenkiel, A. Wibowo, N. Pan, K. Chern, and S. M. Hubbard, Appl. Phys. Lett. 103, 213902 (2013).

${ }^{6}$ A. T. J. van Niftrik, J. J. Schermer, G. J. Bauhuis, P. Mulder, P. K. Larsen, and J. J. Kelly, J. Electrochem. Soc. 154, D629 (2007).
} 
${ }^{7}$ J. J. Schermer, G. J. Bauhuis, P. Mulder, E. J. Haverkamp, J. van Deelen, A. T. J. van Niftrik, and P. K. Larsen, Thin Solid Films 511-512, 645 (2006).

${ }^{8}$ Y. Okada, T. Morioka, K. Yoshida, R. Oshima, Y. Shoji, T. Inoue, and T. Kita, J. Appl. Phys. 109, 024301 (2011).

${ }^{9} \mathrm{~S}$. Tomić, T. Sogabe, and Y. Okada, "In-plane coupling effect on absorption coefficients of InAs/GaAs quantum dots arrays for intermediate band solar cell," Prog. Photovolt. Res. Appl. (published online).
${ }^{10}$ X.-J. Shang, J.-F. He, M.-F. Li, F. Zhan, H.-Q. Ni, Z.-C. Niu, H. Pettersson, and Y. Fu, Appl. Phys. Lett. 99, 113514 (2011).

${ }^{11}$ K. Sablon, J. Little, N. Vagidov, Y. Li, V. Mitin, and A. Sergeev, Appl. Phys. Lett. 104, 253904 (2014).

${ }^{12}$ K. Yoshida, Y. Okada, and N. Sano, J. Appl. Phys. 112, 084510 (2012).

${ }^{13}$ T. Sogabe, Y. Shoji, M. Ohba, K. Yoshida, R. Tamaki, H.-F. Hong, C.-H. Wu, C.-T. Kuo, S. Tomić, and Y. Okada, Sci. Rep. 4, 4792 (2014). 